Artigo Original

\title{
Qualidade do sono em indivíduos com traumatismo raquimedular
}

\section{Sleep quality in subjects with spinal cord injury}

\author{
Rafael Gustavo Alves ${ }^{1}$, Sissy Veloso Fontes², Luciane Bizari Coin de Carvalho ${ }^{3}$, Gilmar \\ Fernandes do Prado ${ }^{4}$
}

\section{RESUMO}

Introdução: Indivíduos com traumatismo raquimedular apresentam diversas alterações em suas vidas. No entanto, a característica do sono desses indivíduos é pouco estudada. Objetivo: Observar a prevalência de distúrbios do sono e a característica do sono em indivíduos com traumatismo raquimedular completo. Método: Os participantes do estudo ( $n=28$ ), 14 com traumatismo raquimedular (Grupo Estudo, GE) e 14 sem (Grupo Controle, GC), responderam a um questionário sobre distúrbios do sono e preencheram diário do sono durante 56 dias. As vaiáveis referentes ao questionário foram analisadas utilizando-se o qui-quadrado e as do diário do sono com o teste-t de Student. Resultados: Dois indivíduos do GE (14\%) apresentaram sono insuficiente, um (8\%) Atraso de Fase, um (8\%) Síndrome das Pernas Inquietas, dois (14\%) Movimentos Periódicos de Membros (PLMS). Esses resultados não foram diferentes dos apresentados pelo GC. O GE mostrou tempo total de sono com 62min a mais $(p=0,03)$, um repouso de 172min a mais $(p=0,01)$ e maior permanência na cama de 85 min a mais $(p=0,02)$ do que o GC. Conclusão: Os indivíduos com traumatismo raquimedular apresentaram mais tempo total de sono, repouso e cochilos em relação ao GC.

\section{Unitermos: Lesões da Medula Espinhal, Sono, Distúrbios do Sono.}

Citação: Alves RG, Fontes SV, Carvalho LBC, Prado GF. Qualidade do sono em indivíduos com traumatismo raquimedular. Rev Neurociencias 2005; 13(4):178-182.

\section{SUMMARY}

Introduction: Individuals with spinal cord injury present many alterations in their lives. The sleep of these people has few studies. Objective: It was to observe the prevalence of sleep disorders and the sleep features of individuals with complete spinal cord injury. Method: 28 participants answered a sleep disorder questionnaire, 14 with spinal cord injury (Study Group, SG) and 14 without (Control Group, CG). A sleep log was completed for 56 days. The variables were analyzed using the Chi Square and t-Student tests. Results: Two individuals of SG (14\%) presented insufficient sleep, one individual (8\%) delayed sleep phase, one individual (8\%) presented restless leg movement syndrome and two individuals (14\%) presented periodic limb movement. These results were not different when compared to the CG. The SG presented total sleep time+nap 62 minutes more, rest 171,59 minutes more,

Trabalho realizado: Universidade Metodista de São Paulo - UMESP, São Paulo, SP

1 - Fisioterapeuta, Universidade Metodista de São Paulo

2 - Fisioterapeuta, Professora de Educação Física, Mestre em Neurociências e Doutora em Ciências pela UNIFESP, Docente da UMESP e UNISANTA

3 - Psicóloga do Setor de Neuro-Sono dos Departamentos de Neurologia e Medicina Baseada em Evidências da Universidade Federal de São Paulo - UNIFESP

4 - Neurologista, Chefe do Laboratório de Sono dos Departamentos de Neurologia e Medicina Baseada em Evidências da Universidade Federal de São Paulo - UNIFESP 
and time in bed 85,36 minutes more than CG. Conclusion: Individuals with spinal cord injury present more total sleep time, rest and naps. However there is no difference in both groups about sleep disorders.

\section{Keywords: Spinal Cord Injuries, Sleep, Sleep Disorders.}

Citation: Alves RG, Fontes SV, Carvalho LBC, Prado GF. Sleep quality in subjects with spinal cord injury. Rev Neurociencias 2005; 13(4):178-182.

\section{INTRODUÇÃO}

O sono desempenha diversas funções que repercutem no bem estar físico e mental dos indivíduos, e na vigília ${ }^{1}$, sendo de extrema importância para as diversas fases do desenvolvimento ${ }^{2}$ e necessário para a manutenção da saúde 3 .

Indivíduos com lesão medular apresentam diversos tipos de alterações e as complicações e seqüelas consistem principalmente em comprometimentos de motricidade, integridade do tônus muscular, sensibilidade, reflexos ${ }^{4}$, provocando alterações autonômicas e interferindo na sexualidade ${ }^{5}$.

A lesão medular provoca bruscas mudanças na vida de um indivíduo, afetando diversos aspectos como físicos, econômicos e sociais, sendo considerado então, um evento devastador ${ }^{6}$.

Dentre os comprometimentos, podemos citar a depressão e a ansiedade ${ }^{7,8} \mathrm{e}$ as complicações respiratórias, como a síndrome da apnéia do sono ${ }^{9-13}$.

As complicações e seqüelas provenientes à lesão medular influenciam significativamente a qualidade de vida e os aspectos relativos ao sono e suas funções.

No entanto, o sono de indivíduos com lesão medular é um assunto pouco encontrado na literatura, portanto estudos quanto a sua qualidade neste estado de morbidade são importantes, pois podem existir aspectos no sono desses pacientes que possam prejudicar a reabilitação.

O objetivo desse estudo foi comparar o padrão de sono e os distúrbios de sono de indivíduos com e sem lesão medular.

\section{MÉTODO}

Foram estudados 14 pacientes com lesão medular traumática (grupo de estudo, GE) e 14 indivíduos sem lesão medular (grupo controle, GC), pareados de acordo com idade e sexo.

Os participantes responderam um questionário sobre Distúrbios do Sono e preencheram um Diário do Sono durante 56 dias.

As variáveis referentes ao questionário de Sono (presença de Distúrbios Respiratórios, Insônia, Avanço de Fase do Sono, Atraso de Fase do Sono, Síndrome das Pernas Inquietas, Movimentos Periódicos das Pernas durante o sono, Sono Insuficiente, Parassonias, Sonolência Excessiva Diurna) foram analisadas utilizando-se o qui-quadrado e as variáveis do diário do sono (Tempo Total de Sono, cochilos, Latência do Sono, tempo acordado após início do sono, repouso, tempo total de cama, eficiência do sono), o teste-t de Student, para comparação dos grupos.

\section{RESULTADOS}

\section{Sono}

Os dados referentes ao diário do sono, nos grupos estudo e controle, são apresentados na Tabela 1: a latência do sono para GE foi de $43,07 \mathrm{~min} \pm 53,1$ e para o GC, 40,59min $\pm 60,15(p=0,90)$. O tempo acordado após o início do sono no GE foi em média 19,85min $\pm 23,64$ e a do GC foi $11,02 \mathrm{~min} \pm 21,08(p=0,3)$. Os cochilos no GE foram de 16,18min $\pm 27,97$, enquanto que no GC foi de $13,73 \mathrm{~min}$ $\pm 22,79(p=0,8)$. E eficiência do sono no GE foi de $85,71 \%$ $\pm 15,75 \%$ e do $G C$ foi de $88,92 \% \pm 9,96 \%$ ( $p=0,52)$.

Tabela 1 . Características do sono para os grupos com lesão medular (GE) e sem lesão medular (GC) avaliados através do diário do sono.

\begin{tabular}{|lccc|}
\hline & Grupo Estudo & Grupo Controle & $p$ \\
\hline Latência do Sono (min) & $43,07 \pm 53,1$ & $40,59 \pm 60,15$ & 0,9 \\
Tempo acordado após início do sono (min) & $19,85 \pm 23,64$ & $11,02 \pm 21,08$ & 0,3 \\
Cochilos (min) & $16,18 \pm 27,97$ & $13,73 \pm 22,79$ & 0,8 \\
Tempo total de sono (min) & $482,64 \pm 100,07$ & $422,75 \pm 57,01$ & 0,06 \\
Tempo total de sono e cochilos (min) & $498,78 \pm 86,38$ & $436,72 \pm 56,68$ & 0,03 \\
Repouso (min) & $248,03 \pm 237,31$ & $76,43 \pm 67,81$ & 0,01 \\
Tempo total de cama (min) & $566,21 \pm 93,67$ & $480,85 \pm 98,85$ & 0,02 \\
Eficiência do Sono (\%) & $85,71 \pm 15,75$ & $88,92 \pm 9,96$ & 0,52 \\
\hline
\end{tabular}


A média do Tempo Total de Sono foi $482,64 \mathrm{~min}$ $\pm 100,07$ para o GE e $422,75 \mathrm{~min} \pm 57,01$ para o CG $(p=0,06)$. A média do Tempo Total de Sono mais cochilos foi de $498,78 \mathrm{~min} \pm 86,38$ e para o GC foi de $436,72 \mathrm{~min} \pm 56,68(p=0,03)$. Repouso para o GE foi de $248,03 \mathrm{~min} \pm 237,31$ e para o GC foi de $76,43 \mathrm{~min}$ $\pm 67,81(p=0,01)$. O tempo total de cama no GE foi de $566,21 \mathrm{~min} \pm 93,67$ e no $\mathrm{GC}$ foi de $480,85 \mathrm{~min}$ $\pm 98,85(p=0,02)$.

\section{Distúrbios de Sono}

Os resultados referentes aos distúrbios do sono são representados na Tabela 2, e nos mostram que nenhum paciente do GE apresentou Distúrbio Respiratório enquanto que $8 \%$ do $G C$ apresentou $(p=0,50)$. No GE, $14 \%$ dos pacientes apresentaram Sono Insuficiente, e nenhum do GC $(p=0,48)$. O Atraso de Fase do Sono esteve presente em $28 \%(p=0,32)$ do GC e em $8 \%$ do GE. Síndrome das Pernas Inquietas esteve presente em $8 \%(p=0,5)$ do GE e nenhuma ocorrência no GC. No GE, Movimentos Periódicos dos Membros durante o Sono foi de $14 \%(p=0,48)$ e nenhum do GC. As variáveis como Sonolência Excessiva Diurna, Narcolepsia, Avanço de Fase do Sono, Insônia e Parassonias não obtiveram nenhuma ocorrência em ambos os grupos.

\section{DISCUSSÃO}

Ao observarmos que o GE apresentou uma tendência a ter um período de sono maior que o GC, notamos que este grupo também apresentou uma predisposição a ficar mais tempo na cama, $85 \mathrm{~min}$ a mais que o GC. Da mesma forma o GE apresentou
Tempo Total de Sono somado aos cochilos $62 \mathrm{~min}$ a mais do que o GC. Dessa forma, mesmo não havendo uma diferença significativa quanto ao tempo de cochilo entre os grupos, houve maior quantidade de cochilos relacionados ao GE. Aparentemente existe uma necessidade ou situação que leva o GE a apresentar mais tempo de repouso $(171,59 \mathrm{~min}$ a mais do que o GC). Pelo diário do sono nota-se que a grande maioria desses indivíduos não possui uma exigência com relação a horários, como no caso de uma atividade laboral ou qualquer outra atividade durante o período da manhã e da tarde. Estudos indicam que a atividade física pode contribuir para minimizar os distúrbios do sono, por influenciarem diretamente o ritmo circadiano, estimulando maior liberação de melatonina, a qual possui relação íntima com o ciclo sono/vigília ${ }^{14}$.

Entretanto, mesmo o GE apresentando o tempo de sono e cochilos superior ao GC, a eficiência do sono foi menor, embora não estatisticamente significante em relação ao GC. Da mesma forma, o tempo de latência do sono e despertares após início do sono não apresentaram diferenças significantes entre os dois grupos. No entanto, observou-se que o GE apresentou maior quantidade de despertares após o início do sono, com uma diferença de 93 despertares a mais em relação ao GC. Não houve questionamentos posteriores que revelassem o verdadeiro motivo desses despertares, o que poderiam estar relacionados com alguma situação referentes à lesão medular como espasticidade, dor neuropática ou depressão. Alguns pacientes podem ter acordado durante o sono para se virarem ou até mesmo para realizar cateterismo.

Quanto aos distúrbios do sono, apenas um indivíduo do GC apresentou distúrbios respiratórios,

Tabela 2. Prevalência (\%) de Distúrbios do Sono para os grupos com lesão medular (GE) e sem lesão medular (GC).

\begin{tabular}{|c|c|c|c|}
\hline & Grupo Estudo & Grupo Controle & $p$ \\
\hline Distúrbio respiratório & 0 & 8 & 0,5 \\
\hline Sonolência excessiva diúrna & 0 & 0 & \\
\hline Narcolepsia & 0 & 0 & \\
\hline Sono insuficiente & 14 & 0 & 0,48 \\
\hline Atraso de fase & 8 & 28 & 0,32 \\
\hline Avanço de fase & 0 & 0 & \\
\hline Insônia & 0 & 0 & \\
\hline RLS & 8 & 0 & 0,5 \\
\hline PLMS & 14 & 0 & 0,48 \\
\hline Parassonia & 0 & 0 & \\
\hline
\end{tabular}


este indivíduo era tabagista por mais de 30 anos e relatou apresentar dificuldades respiratórias. Nenhum paciente do GE apresentou distúrbios nesta categoria, não condizendo com a literatura que refere que, indivíduos com lesão medular, em sua grande maioria, apresentam altos índices de apnéia ou outras complicações respiratórias, inclusive relacionadas ao sono ${ }^{11-13,15}$. No entanto, 9 indivíduos do GE (64\%) manifestaram presença de um sintoma ou situação descrita no questionário sobre distúrbio respiratório, mas que não caracterizou como doença. No GE, 4 indivíduos (28\%) relacionaram problemas como falta de ar e apenas um indivíduo relatou parar de respirar durante o sono. As alterações de problemas respiratórios nesses indivíduos podem variar de acordo com alguns fatores, dentre eles o uso de medicamentos como o Baclofeno e o Benzodiazepínicos (temazepam, diazepam ou nitrazepam) que são utilizados em pacientes com lesão medular, geralmente contra a espasticidade, devido ao efeito de relaxamento muscular ${ }^{12,13,16}$.

Quanto ao Sono Insuficiente, esta variável foi caracterizada no indivíduo que apresentou tempo de sono de 4 a 6 horas por noite. Foi constatado no GE em apenas 2 (14\%) pacientes, com médias de 6 e 3horas respectivamente. Os outros participantes do mesmo grupo tiveram uma média maior que 6 horas de sono por noite. No GC nenhum indivíduo apresentou sono insuficiente. A média diária de sono dos indivíduos do GC foi de 422,48min (7h por noite) enquanto que a do GE foi de $482,31 \mathrm{~min}$ (8h). O Atraso de fase foi maior no GC do que no GE. Nota-se o tempo e a quantidade maior de repouso no período da tarde, resultando inclusive em cochilos, que por sua vez poderiam promover maiores dificuldades para esses indivíduos em dormir no horário habitual, além de não apresentarem nenhum tipo de atividade. Deve-se lembrar que a grande maioria dos participantes do GE fazia o uso de medicamentos e somando com a falta de atividade, ambos exerciam influencias sobre o ritmo circadiano e conseqüentemente no sono, provocando o atraso de fase nestes indivíduos.

\section{CONCLUSÃO}

Não houve diferença entre a quantidade de indivíduos que apresentaram qualquer distúrbio do sono nos GE e GC, porém constamos que indivíduos com traumatismo raquimedular apresentaram períodos maiores de sono, permaneceram mais tempo na cama, cochilam mais vezes e por mais tempo e tiveram mais períodos de repouso do que os indivíduos do GC.

\section{REFERÊNCIAS BIBLIOGRÁFICAS}

1. Ceresér CC, Weidlich J, Martinez D, Zorzi L, Manoela JC. Sono: qual é a sua funçäo? Sleep functions. Rev med PUCRS 1999; 9: 257-61.

2. Roth T. Characteristics and Determinants of Normal Sleep. J Clin Psychiatr 2004; 65: 8-11.

3. Dogan O, Ertekin S, Dogan S. Sleep quality in hospitalized patients. J Clin Nurs 2005; 14: 107-113.

4. Machado ABM; Neuroanatomia Funcional. Rio de Janeiro: Atheneu; 1993.

5. Ahn SH, Park HW, Lee BS, et al. Gabapentin effect on neurophatic pain compared among patients with spinal cord injury and different durations of symptoms. Spine 2003; 28: 341-46.

6. Freed MM. Lesões Traumáticas e Congênitas da Medula Espinhal. In: Kotte FJ et al. (eds). Tratado de Medicina
Física e Reabilitação de Krusen. V2, $4^{\circ}$ ed. São Paulo: Manolo, 1994; 709-38.

7. Carvalho SAD, Andrade MJ, Tavares MA, Freitas JLS. Spinal cord injury and psycological response. GenHosp Psychiatr 1998; 20: 353-59.

8. Bombardier $\mathrm{CH}$, Richards JS, Krause JS, Tulsky D, Tate DG. Symptoms of major depression in people with spinal cord injury: Implications for screening. Arch Phys Med Rehabil 2004; 85: 1749-1756.

9. Pereira VC, Fontes VF, Perez AJ, Fukujima MM. Protocolo de Tratamento Fisioterápico da Dinâmica Respiratória em Pacientes com Lesão Medular completa em Diferentes Níveis Cervicais e Torácico Baixo. Rev Neurociências 1998; 6: 81-85.

10. Biering-Sorensen F, Biering-Sorensen M. Sleep distur- 
bances in the spinal cord injured: an epidemiological questionnaire investigation, including a normal population. Spinal Cord 2001; 39:505-513.

11. Burns SP, Little JW, Hussey JD, Lyaman P, Lakshminarayanan S. Sleep Apnea Syndrome in Chronic Spinal Cord Injury: Associated Factors and Treatment. Arch Phys Med Rehabil 2000; 81: 1334-1339.

12. Berlowitz DJ, Brown DJ, Campbell DA, Pierce RJ. A Iongitudinal evaluation of sleep and breathing in the first year after cervical spinal cord injury. Arch Phys Med Rehabil 2005; 86:1193-1199.

13. Stockhammer E, Tobon A, Michel F, et al. Characteristics of sleep apnea syndrome in tetraplegic patients. Spinal Cord Injury 2002; 40:286-294.

14. Mello MT, Boscolo RA, Esteves AM, Tufik S. O exercício físico e os aspectos psicobiológicos. Rev Bras Med Esporte 2005; 11:203-207.

15. Wang TG, Wang YH, Tang FT, Lin KH, Lien IN. Resistive Inspiratory Muscle Training in Sleep-Disordered Breathing of Traumatic Tetraplegia. Arch Phys Med Rehabil 2002; 83: 491-496.

16. Broderick CP, Radnitz CL, Bauman WA. Diazepam usage in veterans with spinal cord injury J Spinal Cord Med 1997; 20: 406-409. 